\title{
Out-of-home Activity Accessibility Checklist for Parents of Children with Gross Motor Dysfunction
}

\author{
Asuka Kenmochi ${ }^{1}$, Chieko Karashima ${ }^{2}$ \\ ${ }^{1}$ Aoi-tori Plus (Child development support centre), Aoi Gakuen School Corporation, Japan \\ ${ }^{2}$ Department of Physical and Occupational Therapy, Graduate School of Medicine, Nagoya University, Japan
}

\begin{abstract}
Background: Parents of children with gross motor dysfunction often face obstacles when performing out-ofhome activities with their children. However, little is known concerning their situation.

Objective: This study aimed to develop an out-of-home activity accessibility checklist for parents of children with gross motor dysfunction that clarifies their situation during these activities.

Method: Parents of children with gross motor dysfunction participated in semi-structured interviews to generate a tentative accessibility checklist. Then, Delphi questionnaire survey was conducted to determine appropriate checklist items.

Result: Fifteen participants involved in the semi-structured interview and 124 tentative checklist items were generated. Then, 56 participants involved in Delphi questionnaire survey and tentative checklist items was reduced to 39 . These checklist items were grouped under the domains of obstacles, coping strategies, and required assistance and resources. Most obstacles were caused by the lack of equipped infrastructures.

Discussion: Most obstacles faced by parents of children with gross motor dysfunction were caused by the lack of infrastructures that were barrier-free. The parents had to collect extensive information prior to outings as coping strategies. Therefore, parents require society level environment improvement in order to participate in out-of-home activities. The checklist may provide local communities with valuable information to construct more accessible environments and inclusive communities.
\end{abstract}

Keywords: gross motor dysfunction, family support, social participation, parents

(Asian J Occup Ther 16: 63-69, 2020)

\section{Introduction}

Children with gross motor dysfunction face various obstacles when participating in out-of-home activities; activities performed outside the home such as leisure activities and IADLs, compared with children without. Furthermore, their parents may face additional obstacles during outings with their children.

There are several factors that would limit the participation of children from out-of-home activities such as gross motor dysfunction [1], maladaptive behaviours [1], family relationships [2], parent fatigue [3, 4], unsuitable

Received: 4 October 2019, Accepted: 22 April 2020

Corresponding to: Asuka Kenmochi, Aoi-tori Plus (Child development support centre), Aoi Gakuen School Corporation, 4-12, West 11, South 17, Obihiro, Hokkaido 080-0021, Japan

e-mail:kenmochiasuka@gmail.com

(C)2020 Japanese Association of Occupational Therapists environments [3, 5-7], and attitude and behaviours of the general public [4-6, 8]. Previous studies mostly examined the participation in out-of-home activities that were focused on leisure or school activities.

In terms of environmental assessment, many accessibility checklists had been developed and applied [9-11]. These checklists aimed to evaluate how a community, specific place, event and activity etc. accessible for people with disabilities to generate inclusive environment. Therefore, these checklist items did not reflect their individual factors that disturb their participation.

The participation in out-of-home activities is also limited for parents when they go out together with their children who have gross motor dysfunction. For example, Davis et al. (2010) indicated that some families of children with gross motor dysfunction gave up leisure activities, such as family trips, due to difficulties in finding convenient barrier-free destinations. In addition to leisure activities, parents may need to go out with their 
children for instrumental activities of daily living (IADL) such as shopping, financial management, or child rearing [12].

Currently, it is unclear what specific obstacles parents of children with gross motor dysfunction encounter in social settings of IADLs compared with leisure activities. Therefore, the purpose of this study was to establish an out-of-home activity accessibility checklist for these parents to document their obstacles, coping strategies, and required resources when they perform out-of-home activities with their children.

\section{Method}

\section{Study design}

The Delphi technique, an established method to develop diagnostic criteria or new evaluation tools for diseases and medical conditions, was used to determine the appropriate checklist items to evaluate the out-ofhome activity accessibility for parents of children with gross motor dysfunction $[13,14]$. In this study, parents were recruited if they (a) had children with gross motor dysfunction; children with physical disability (e.g., cerebral palsy, spina bifida, etc.) and that affect their mobility aged between two and 18 years, (b) were the primary caregiver, (c) had children who lived with them, and (d) had children who, at least once a month, visited a paediatrician, occupational, or physical therapist at our cooperating institutions.

The Delphi questionnaire was developed using the following process that would establish the out-ofhome activity accessibility checklist items. First, faceto-face semi-structured interviews were conducted with some participants. The transcribed data were analysed to generate a tentative accessibility checklist. Through a revision process, the first-round Delphi questionnaire was developed by assessing the appropriateness of each tentative checklist item. Other participants were asked to answer the three rounds Delphi questionnaires in order to reach a consensus in the tentative items by rating them on a five-rank Likert Scale. Finally, the accessibility checklist items to be used were obtained from those that achieved a consensus of $\geq 80 \%$. The protocol for the research project was approved by the Ethical Committee of the university. Informed consent was obtained from all participants prior to data collection.
Procedure to establish the out-of-home activity accessibility checklist items

\section{Qualitative study to formulate first-round Delphi ques- tionnaire}

Step 1. Generate tentative out-of-home activity accessibility checklist items

Participants residing in the central and west-central region of Japan participated in this process between October 2014 and March 2015. Face-to-face semi-structured interviews were conducted to explore any possible items for the accessibility checklist. By considering concrete examples of outings for IADLs and leisure activities, the participants answered the following questions: obstacles encountered, coping strategies, and forms of assistance or resources that were required when they went out with their children with gross motor dysfunction. The first author of this paper, who carried out the interviews, was not associated with the collaborative institutions or the study participants.

Each interview was audio-recorded with the participants' consent and transcribed verbatim. The entire transcript was cut into minimal meaning units, and the units were deductively divided into the domains of obstacles, coping strategies, and required assistance and resources. Then, inductive analysis [15] was taken to discern similarities among the meaning units. Similar meaning units were grouped together and categories were developed by naming these groups. These categories created the tentative accessibility checklist items. After the analysis for both IADLs and leisure activities were finished, the tentative accessibility checklist items were examined whether they were specific for IADLs, leisure activities, or were a common feature to both. Both of the authors were involved in the entire analysis process.

\section{Step 2. Develop first-round Delphi questionnaire con- sisting of the tentative items}

The tentative accessibility checklist items from both the obstacles and coping strategies domains were sorted by three processes: planning and preparing for the outing, transit, and performance of the aimed activities at the destination. Next, the prototype firstround Delphi questionnaire was developed. A five-rank Likert Scale was applied to examine how appropriate the tentative items were as an out-of-home activity accessibility checklist for the parents of children with gross motor dysfunction using a range from one (definitely inappropriate) to five (definitely appropriate). The participants residing in the central region of Japan were involved in the revision process for the first-round Delphi questionnaire prototype between July and August 2016. They were asked to answer the first-round Delphi 
questionnaire prototype and to give their opinions regarding the ambiguity of the questionnaire items, any issues regarding answering the questionnaire items, the layout of the questionnaire, and any further comments or suggestions. Based on their responses, the first-round Delphi questionnaire was revised and finalized.

\section{Delphi questionnaire survey}

A different set of participants living in western Japan participated in the Delphi questionnaire survey to assess the accessibility checklist items. The survey was iterated three times (Hsu et al., 2007) and was given once every three months between September 2016 and May 2017. The authors sent each round of the questionnaire to the collaborating institutions, where the staff provided the questionnaires to the participants. After the participants filled out the questionnaire, they were collected and sent back to the authors. In the firstround survey, the participants answered the first-round Delphi questionnaire developed through the qualitative study. During the second and third-round surveys, the participants who participated in the previous surveys reassessed the same questionnaire items but also took into account the score distribution from the previous questionnaire that was shown by a band chart. Some items of the questionnaire in the previous rounds were reassessed, modified, or newly added based on the opinions from the open-ended comment box in the previous surveys. The questionnaire also asked the participants for information regarding themselves and their child (sex, age, diagnosis, etc.). The tentative accessibility checklist items were narrowed down to those that provided concise information associated with the performance of out-of-home activities of parents of children with gross motor dysfunction. A consensus was defined as $\geq 80 \%$ agreement as determined by the results from the third-round survey $[16,17]$.

\section{Results}

\section{Qualitative study to formulate first-round Delphi ques- tionnaire}

Step 1. Generate tentative out-of-home activity accessibility checklist items

Fifteen participants involved in the semi-structured interview, and the data of 14 participants were analysed. One participant data was excluded because she could not answer all of the questions. The average length of the interviews was $31 \pm 9$ (range, 13-45) minutes. All the participants were female (mothers) and were $42 \pm$ 5 (range, 31-48) years old. Their children were $6 \pm 5$ (range, 2-16) years old of which 11 had cerebral palsy (including periventricular leukomalacia, PVL), two had spina bifida, and one had chromosomal abnormalities. A total of 124 tentative checklist items were generated by the qualitative procedure from step 1 . Of the tentative items, 80, 34, and 10 were classified in the obstacles, strategies, and required assistance and resources domains, respectively.

\section{Step 2. Develop first-round Delphi questionnaire con- sisting of the tentative items}

Three participants participated in a revision process. Based on their feedback, the tentative checklist items were reconsidered, modified, or integrated. As a result, the total number of tentative items was reduced to 115 . Of these, 72, 34, and nine were classified into the obstacles, strategies, and required assistance and resources domains, respectively. The revised list of items formed the first-round Delphi questionnaire.

\section{Delphi questionnaire survey}

Forty-three of 56 participants (77\%) completed three rounds of the iterative survey (Table 1). Twentyfour and six new items were added to the second-round and third-round questionnaire, respectively. As a result, a total of 145 tentative items were examined in the thirdround questionnaire survey. In the third-round questionnaire survey, the average age of the participants was 41 \pm 5 (range, 31-50) years, and the age of their children was $8 \pm 5$ (range, 2-18) years. Of these children, 21 (48\%) had cerebral palsy (including PVL, infantile encephalitis, etc.), 10 (23\%) had anomalies of the central nervous system (including spina bifida, hydrocephalus, etc.), eight (18\%) had chromosomal abnormalities, one ( $2 \%$ ) had developmental motor retardation, and five (11\%) had no described conditions. Thirty-seven of 44 (84\%) children required assistance to move around outdoors to some degree. Since 39 of the 145 (27\%) items had a consensus of $80 \%$ or greater, they were adopted into the accessibility checklist (Table 2).

\section{Discussion}

This study developed an out-of-home activity accessibility checklist items for the parents of children with gross motor dysfunction. The checklist consisted of 39 items to obtain information regarding the process of out-of-home activities in terms of obstacles, coping strategies, and required resources.

Similar to previous studies [3, 4, 18, 19], most of the study participants were mothers even though the inclusion criterion was primary caregivers. In the qualitative study process, $57 \%$ of children required assistance to move outside to some degree and, of these, $71 \%$ had 
Table 1 Demographic characteristics of subjects and their children with gross motor dysfunction

\begin{tabular}{|c|c|c|c|}
\hline Round & 1 & 2 & 3 \\
\hline \multicolumn{4}{|c|}{ Subjects } \\
\hline \multicolumn{4}{|l|}{ Gender } \\
\hline Female & 51 & 45 & 39 \\
\hline Male & 1 & 1 & 1 \\
\hline Not described & 3 & 3 & 3 \\
\hline Age (years) & $40 \pm 5$ & $41 \pm 5$ & $41 \pm 5$ \\
\hline \multicolumn{4}{|c|}{ Children $^{\mathrm{a}}$} \\
\hline \multicolumn{4}{|l|}{ Gender } \\
\hline Female & 32 & 29 & 25 \\
\hline Male & 23 & 20 & 18 \\
\hline Not described & 1 & 1 & 1 \\
\hline Age (years) & $7 \pm 5$ & $8 \pm 5$ & $8 \pm 5$ \\
\hline \multicolumn{4}{|l|}{ Diagnosis } \\
\hline Cerebral palsy $^{\mathrm{c}}$ & 31 & 31 & 21 \\
\hline Nervous system deformity ${ }^{\mathrm{d}}$ & 10 & 5 & 10 \\
\hline Chromosome abnormality & 9 & 9 & 8 \\
\hline Others & 2 & 0 & 1 \\
\hline Not described & 5 & 6 & 5 \\
\hline \multicolumn{4}{|l|}{ Assistance with outdoor mobility } \\
\hline Requires assistance & 47 & 41 & 37 \\
\hline Independent & 7 & 7 & 5 \\
\hline Not described & 2 & 2 & 2 \\
\hline \multicolumn{4}{|l|}{ Use of facilities } \\
\hline Developmental support centres ${ }^{\mathrm{e}}$ & 20 & 15 & 10 \\
\hline Special classrooms & 6 & 6 & 4 \\
\hline Special schools & 24 & 24 & 24 \\
\hline
\end{tabular}

Note. ${ }^{\text {a }}$ The number of subjects and their children are not the same due to the inclusion of one parent of twins with movement disorders.

${ }^{\mathrm{b}}$ Multiple answers were allowed under the diagnosis category.

${ }^{\mathrm{C}}$ Cerebral palsy included periventricular leukomalacia, infantile encephalitis, etc.

${ }^{\mathrm{d}}$ Nervous system deformity included spina bifida, hydrocephalus, etc.

${ }^{\mathrm{e}}$ Developmental support centres are facilities that provide medical treatment and education for pre-school children with disabilities.

cerebral palsy. In the third-round Delphi questionnaire survey, $83 \%$ of children required assistance to move outside to some degree and, of these, $48 \%$ had cerebral palsy. The Japanese Ministry of Health, Labour and Welfare (2008) reported that $83 \%$ of children with gross motor dysfunction required assistance to move outside to some degree, and, of these, $48 \%$ had cerebral palsy. Therefore, the third-round Delphi questionnaire survey participants represented the children with gross motor dysfunction in Japan well. However, there was a discrepancy in distribution of disorders and severity between the qualitative study process and questionnaire survey participants, and this may lead to a large increase in number of tentative checklist items in the second-round questionnaire survey.

This study focused on the accessibility for parents of children with gross motor dysfunction when they perform out-of-home activities in terms of IADLs and leisure activities. However, the parents take care of their children whenever they go out with their children even though they perform leisure activities. In particular, parents collected extensive information regarding places they could visit for leisure activities, such as family trips or dining (\#28-32), prior to their outing. The results from this study also indicated that some gave up these activities when they felt inconvenienced by their children based on the destination information and experiences from the past $(\# 2,3)$. In addition, the parents had more care burdens during prolonged outings for leisure activities since they involved feeding (\#21), bathing (\#25-27) as well as washroom assistance (\#17-19). Previous studies also showed that prior unfavourable experiences due to unsuitable environments and attitudes and behaviours of the general public negatively affected family leisure activities [3, 4].

In the obstacles domain, there were two types of checklist items: individual level items relating to children (e.g. \#1, 2) and families (e.g. \#15, 20), and society level items relating to environment (e.g. \#5-12) and social attitude (e.g. \#3, 4, 13). The majority of items (23 items) were society level items. Several studies have also reported that unsuitable environments and attitudes and behaviours of the general public limited the participation of children with disabilities and their families $[3,4,6,8,21]$. Furthermore, all the items established in the required assistance and resources domain required society level improvement (\#35-39). These results indicate that many obstacles were due to physical and social environments and that society level improvement is required to overcome these challenges. Recently, the number of occupational therapists involved in community development is increasing [22]. Since occupational therapists are unique professionals with diverse knowledge regarding medical disabilities and environment evaluation [23], occupational therapists may help in encouraging families of children with gross motor dysfunction to participate in out-of-home activities through their involvement in community environment coordination, educational activities to enhance the understanding of disabilities to the public as well as their direct intervention with children.

Limitations to our study may include the lack of certain background information of participants such as severity of their child gross motor dysfunction, major mode of transport, marital status, employment status, socioeconomic status, and educational background. This information may have affected the outcome of our study. In addition, there is no existing nationwide statistical data with this type of caregiver information to compare 
Table 2 Consensus ratio and characteristics of the determined checklist items $(n=43)$.

\begin{tabular}{|c|c|c|c|}
\hline \multirow{2}{*}{ Questionnaire item } & \multirow{2}{*}{$\begin{array}{c}\text { Consensus } \\
\text { ratio } \\
(\%)\end{array}$} & \multicolumn{2}{|c|}{$\begin{array}{l}\text { Category } \\
\text { classification }\end{array}$} \\
\hline & & Purpose $^{\mathrm{a}}$ & Factor $^{\mathrm{b}}$ \\
\hline \multicolumn{4}{|l|}{ Obstacles domain } \\
\hline \multicolumn{4}{|l|}{ 1. Planning and preparing for outings } \\
\hline \#1 Restriction in the type of destination due to weather & 95 & $\mathrm{C}$ & I \\
\hline \#2 Hesitation or resignation of outing due to lack of human resources for child care & 81 & $\mathrm{~L}$ & I \\
\hline $\begin{array}{l}\text { \#3 Few or no appropriate places for children with disabilities to play safely } \\
\text { (e.g., environment/other children) }\end{array}$ & 93 & $\mathrm{~L}$ & $\mathrm{~S}$ \\
\hline \multicolumn{4}{|l|}{ 2. Transit } \\
\hline \#4 Lack of parking for those with disabilities (e.g., insufficient number/misuse by general public) & 83 & $\mathrm{C}$ & $\mathrm{S}$ \\
\hline \#5 Lack of space to move wheelchairs in parking lot & 88 & $\mathrm{C}$ & $\mathrm{S}$ \\
\hline \#6 No roofs over parking lot & 95 & $\mathrm{C}$ & $\mathrm{S}$ \\
\hline \#7 No elevators at station & 86 & $\mathrm{C}$ & $\mathrm{S}$ \\
\hline \#8 Roundabout route to elevator & 86 & $\mathrm{C}$ & $\mathrm{S}$ \\
\hline \#9 Complicated layout of large stations & 84 & $\mathrm{C}$ & $\mathrm{S}$ \\
\hline \#10 Large number of stairs at subway stations & 84 & $\mathrm{C}$ & $\mathrm{S}$ \\
\hline \#11 Long distance between entrance gate to boarding area at stations & 81 & $\mathrm{C}$ & $\mathrm{S}$ \\
\hline \#12 Large gap between train and platform & 88 & $\mathrm{C}$ & S \\
\hline \#13 Worries in moving around crowded stations by wheelchair (e.g., attitude of general public) & 81 & $\mathrm{C}$ & S \\
\hline \#14 Uneven footpaths & 81 & $\mathrm{C}$ & $\mathrm{S}$ \\
\hline \#15 Difficulty in using umbrellas while pushing wheelchair & 91 & $\mathrm{C}$ & I \\
\hline \multicolumn{4}{|l|}{ 3. Performance of aimed activities at destination } \\
\hline \#16 Long wait times for crowded elevators (e.g., insufficient number/ thoughtlessness of general public) & 81 & $\mathrm{C}$ & $\mathrm{S}$ \\
\hline $\begin{array}{l}\text { \#17 Lack of equipped restrooms for people with disabilities } \\
\text { (e.g., insufficient number/misuse by general public) }\end{array}$ & 86 & $\mathrm{C}$ & $\mathrm{S}$ \\
\hline \#18 Lack of space to move wheelchairs in restroom & 88 & $\mathrm{C}$ & $\mathrm{S}$ \\
\hline \#19 No appropriate change tables for people with disabilities & 95 & $\mathrm{C}$ & S \\
\hline \#20 Difficulty in handling both shopping cart and wheelchair at grocery stores & 91 & $\mathrm{P}$ & I \\
\hline \#21 No appropriate chairs for children with disabilities at restaurants & 84 & $\mathrm{~L}$ & $\mathrm{~S}$ \\
\hline \#22 Rough walkways at tourist sites & 93 & $\mathrm{~L}$ & $\mathrm{~S}$ \\
\hline \#23 Few equipped attractions for people with disabilities at amusement parks & 86 & $\mathrm{~L}$ & $\mathrm{~S}$ \\
\hline \#24 Narrow entrance gates for wheelchairs & 84 & $\mathrm{~L}$ & $\mathrm{~S}$ \\
\hline \#25 Slippery floors at pools/public baths & 88 & $\mathrm{~L}$ & S \\
\hline \#26 Limited space to take care of child at pools/public baths & 91 & $\mathrm{~L}$ & $\mathrm{~S}$ \\
\hline $\begin{array}{l}\text { \#27 Difficulty in taking adolescents of the opposite sex to pools/public baths } \\
\text { (e.g., environment/social stigma) }\end{array}$ & 88 & $\mathrm{~L}$ & $\mathrm{~S} / \mathrm{I}$ \\
\hline
\end{tabular}

Coping strategies domain

1. Planning and preparing for outings

28 Plan to re-visit familiar facilit

\#29 Reserve a private room or other appropriate environment for dining out

\#30 Check facility for barrier-free access (e.g., ramps and elevators)

\#31 Check availability of washrooms for those with disabilities

\#32 Check discount information for people with disabilities

(i.e., if the facility has a discounted entrance fee, it may be equipped for people with disabilities)

3. Performance-aimed activities at certain places

\#33 Use of a shoulder bag or backpack to take care of their children

\#34 Bring children's extra clothes

$\begin{array}{ll}81 & \text { L } \\ 86 & \text { L } \\ 84 & \text { L } \\ 81 & \text { L } \\ 86 & \text { L }\end{array}$

Required assistance and resources domain

\#35 Improve restroom environment (e.g., include change tables for people with disabilities)

\#36 Equip rooms for rest/meals etc. for people with disabilities

\#37 Improve footpaths, and reduce the number of barriers, stairs, and potholes

\#38 Implement footpaths with roofs

\#39 Increase parking area for people with disabilities

$\begin{array}{ll}86 & \mathrm{C} \\ 88 & \mathrm{C}\end{array}$

Note. ${ }^{\text {a }}$ Classification by purpose of out-of-home activities. C: common items for both IADL and leisure activities. P: particular items for IADLs such as grocery shopping, dropping off and picking up children from school, etc. L: particular items for leisure activities such as family trips, etc.

${ }^{\mathrm{b}}$ Classification by level of obstacles: S, society level; I, individual level. 
with the participants in this study. Furthermore, although the purpose of this study was to clarify the accessibility checklist items for both parents, most of the study participants were mothers. Therefore, several checklist items reflecting the opinion of fathers should be added in future studies.

\section{Conclusion}

To our knowledge, this study is the first to clarify the accessibility of out-of-home activities from the perspective of parents since many prior studies were conducted through the perspective children. Most of the obstacles encountered by parents during outings with their children with gross motor dysfunction came from society level issues such as lack in barrier-free infrastructures. Prior to going out for leisure activities, parents would have to collect extensive information regarding their destination in order to mitigate issues during their outing. Based on these results, parents require society level improvements, and the checklist may provide local communities with valuable information to construct more accessible environments and inclusive communities. Further studies are necessary to develop a more practical use for this accessibility checklist.

\section{Acknowledgements}

The authors would like to acknowledge the time and effort of all participants, cooperators of associated facilities for this study.

\section{Conflicts of Interest}

The authors declare that there are no conflicts of interest regarding the publication of this paper.

\section{References}

[1] Schreuer N, Sachs D, Rosenblum S. Participation in leisure activities: Differences between children with and without physical disabilities. Res Dev Disabil. 2014; 35(1): 223-33.

[2] Chiarello LA, et al. Determinants of participation in family and recreational activities of young children with cerebral palsy. Disabil Rehabil. 2016; 38(25): 2455-68.

[3] Davey H, Imms C, Fossey E. 'Our child's significant disability shapes our lives': experiences of family social participation. Disabil Rehabil. 2015; 37(24): 2264-71.

[4] Davis E, et al. The impact of caring for a child with cerebral palsy: quality of life for mothers and fathers. Child Care Health Dev. 2010; 36(1): 63-73.

[5] Dehghan L, Dalvandi A, Rassafiani M, Hosseini SA, Dalvand H, Baptiste S. Social participation experiences of mothers of children with cerebral palsy in an Iranian context. Aust Occup Ther J. 2015; 62(6): 410-9.

[6] Mei C, et al. Activities and participation of children with cerebral palsy: parent perspectives. Disabil Rehabil. 2015; 37(23): 2164-73.

[7] Piškur B, Beurskens AJHM, Jongmans MJ, Ketelaar M, Smeets RJEM. What do parents need to enhance participation of their school-aged child with a physical disability? A cross-sectional study in the Netherlands. Child Care Health Dev. 2015; 41(1): 84-92.

[8] Vogts N, Mackey AH, Ameratunga S, Stott NS. Parentperceived barriers to participation in children and adolescents with cerebral palsy. J Paediatr Child Health. 2010; 46(11): 680-5.

[9] Dolbow DR, Figoni SF. Accommodation of wheelchairreliant individuals by community fitness facilities. Spinal Cord. 2015; 53(7): 515-9.

[10] Doshi JK, Furlan AD, Lopes LC, DeLisa J, Battistella LR. Conferences and convention centres' accessibility to people with disabilities. J Rehabil Med. 2014; 46(7): 616-9.

[11] Calder AM, Mulligan HF. Measurement properties of instruments that assess inclusive access to fitness and recreational sports centers: a systematic review. Disabil Health J. 2014; 7(1): 26-35.

[12] American Occupational Therapy Association, Occupational therapy practice framework: Domain and process (3rd Ed.), vol. 68, no. Suppl. 1. Bethesda: American Occupational Therapy Association, Inc., 2014.

[13] Hasson F, Keeney S, McKenna H. Research guidelines for the Delphi survey technique. J Adv Nurs. 2000; 32(4): 1008-15.

[14] Hsu C-C, Brian S. The Delphi Technique: Making Sense of Consensus - Practical Assessment, Research \& Evaluation. 2007; 12(10).

[15] Patton MQ. Qualitative research \& evaluation methods: integrating theory and practice, 4th editio. Thousand Oaks, California: SAGE Publications, 2015.

[16] Bond KS, Jorm AF, Kitchener BA, Kelly CM, Chalmers KJ. Development of guidelines for family and nonprofessional helpers on assisting an older person who is developing cognitive impairment or has dementia: a Delphi expert consensus study. BMC Geriatr. 2016; 16(1): 129.

[17] Ross AM, Kelly CM, Jorm AF. Re-development of mental health first aid guidelines for suicidal ideation and behaviour: a Delphi study. BMC Psychiatry. 2014; 14(1): 241.

[18] Al-Gamal E, Long T. Psychological distress and perceived support among Jordanian parents living with a child with cerebral palsy: a cross-sectional study. Scand J Caring Sci. 2013; 27(3): 624-31.

[19] LaForme Fiss A, et al. Family ecology of young children with cerebral palsy. Child Care Health Dev. 2014; 40(4): 562-71.

[20] Ministry of Health Labour and Welfare, "Survey on persons with pyhsical disability," 2008. [Online]. Available: 
http://www.mhlw.go.jp/toukei/itiran/eiyaku.html. [Accessed: 06-Jun-2017].

[21] Piškur B, et al. The lived experience of parents enabling participation of their child with a physical disability at home, at school and in the community. Disabil Rehabil. 2016; 38(8): 803-12.

[22] Leclair LL, Ashcroft ML, Canning TL, Lisowski MA.
Preparing for community development practice: A Delphi study of Canadian occupational therapists. Can J Occup Ther. 2016; 83(4): 226-36.

[23] McClain L, Lutz J, Salmans D, Wright S. Shopping Mall Wheelchair Accessibility Checklist Based on the ADA Guidelines. Occup Ther Heal care. 1999; 11(4): 41-66. 